ISSN electrónico: 2172-9077

DOI: https://doi.org/10.14201/fjc-v22-25766

\title{
TENDENCIAS E IMPACTO DE LA INTELIGENCIA ARTIFICIAL EN COMUNICACIÓN: COBOTIZACIÓN, GIG ECONOMY, CO-CREACIÓN Y GOBERNANZA
}

\section{Trends and impact of artificial intelligence in communication: cobotisation, gig economy, co-creation and governance}

\author{
Dr. José Miguel TÚÑ̃Z LÓPEZ \\ Profesor de la Universidade de Santiago de Compostela, España \\ miguel.tunez@usc.es \\ (iD) https://orcid.org/0000-0002-5036-9143
}

Fecha de recepción del artículo: 12/02/2021

Fecha de aceptación definitiva: 18/02/2021

\begin{abstract}
RESUMEN
Este artículo revisa la producción científica sobre el impacto de la tecnología en la gestión de la comunicación, de un modo global, y de la identificación de cambios y tendencias por la aplicación de la IA, de modo más concreto. Es una investigación interpretativa en la que se trabaja sobre una muestra intencional creada a partir de resultados de búsqueda en Google Scholar, WoS y Scopus, acotados a un periodo longitudinal de cinco años (2016 a 2020). Se realiza una evaluación interpretativa de textos a partir de preguntas abiertas para las que se busca una respuesta no predeterminada. Los resultados definen un panorama marcado indefiniciones semánticas y transversalidad e hibridación de campos. Se destaca el impacto en la gobernanza, la cobotización, la co-creación de contenidos y en, entre otros, aspectos éticos y jurídicos. La revisión bibliográfica evidencia que están cambiando los roles porque se transforman los escenarios y las funciones, con nuevas actitudes y nuevos conceptos. La hibridación de soportes se traslada a la hibridación de perfiles y de necesidades (cobotización), la gobernanza de la IA está en construcción y los ajustes en rutinas y perfiles demandan menos operatividad y más estrategia.
\end{abstract}

Palabras clave: Inteligencia artificial; gestión de la comunicación; gig economy; gobernanza, co-participación

\begin{abstract}
This article reviews the scientific production on the impact of technology on communication management, in a global way, and the identification of changes and trends due to the application of AI, in a more concrete way. It is an interpretative research that works on a purposive sample created from search results in Google Scholar, WoS and Scopus, limited to a longitudinal period of five years (2016 to 2020). An interpretative evaluation of texts is carried out on the basis of open questions for which a non-predetermined answer is sought. The results define a panorama marked by semantic indefinitions and transversality and hybridisation of fields. The impact on governance, cobotisation, co-creation of content and, among others, ethical and legal aspects are highlighted. The literature review shows that roles are changing because scenarios and functions are being transformed,
\end{abstract}


with new attitudes and new concepts. The hybridisation of media channels moves to the hybridisation of profiles and needs (cobotisation), AI governance is under construction and adjustments in routines and profiles demand less operability and more strategy.

Keywords: Artificial intelligence; communication management; gig economy; governance; co-participation.

\section{Introducción}

La Inteligencia Artificial (IA) se ve como una consecuencia del avance de la tecnología que se enmarca en la llamada Cuarta Revolución Industrial, a la que se confiere un carácter de impacto sistémico que se amplía a transformaciones globales porque se extiende, por igual, a la vida social, política y económica. No está exenta de controversia, incluso desde su propio campo científico, con voces que aseguran que el avance real está en los datos y en su procesamiento más que en las técnicas que se aplican porque "aún se desconocen muchos conceptos básicos de inteligencia y cognición". "Se está discutiendo mucho, con visiones del futuro entre el apocalipsis y la utopía" (Krischh, 2020) como IA e Internet afectan a la plasticidad del cerebro y modifican nuestros mapas neuronales para proponer soluciones ya tomadas que eviten ejercitar el pensamiento (Carr, 2011).

En la última década se ha registrado un incremento exponencial de la atención científica sobre el impacto de la IA, no solo en sus propios campos computacionales o robóticos, sino en todas las disciplinas en la que se van incorporando sus aplicaciones. Es el contrapunto a etapas anteriores de apenas actividad de divulgación científica. Belda relata que, entre 1970 y 1985, la IA "se vio sumida en un largo invierno de producción científica" provocado por los problemas que se encontraron "las dos aproximaciones complementarias en las que se había estructurado la comunidad académica: la top-down y la bottom-up" (2019, p. 85). El resurgir dura hasta el final de la guerra fría, al inicio de la última década del paso siglo XX, en la que la IA entra de nuevo en una etapa de baja actividad. "Fue una suerte de calma antes de la tempestad, entendiendo esa tempestad como el avance final que ha provocado que hoy en día la inteligencia artificial, probablemente sin saber este gobernando prácticamente todos los aspectos de nuestra vida cotidiana" (Belda: 2019, p. 81).

La IA se vincula a la computación y la informática, aunque el concepto basal es la lógica aristotélica porque se fundamenta en silogismos, es decir, en razonamientos deductivos derivados de dos proposiciones a modo de premisas entre las que se establece una inferencia deductiva para extraer una tercera, o conclusión.

Casi todos los trabajos sobre IA obvian cualquier referencia anterior a Turing. El camino recorrido, sin embargo, se remonta a las contribuciones de Aristóteles (384 aC-322 a.C.) y de Ctesibio de Alejandría (285 a.C.-222 a.C.), que construyó la primera máquina autocontrolada que funcionaba siguiendo instrucciones incorporadas con antelación por lo que podría considerarse el primer algoritmo en la historia. "Mientras el primero inventó la lógica primitiva, al otro se le atribuye la algorítmica" (Belda: 2019, p. 17).

Los tratados de IA señalan a Boole y De Morgan como transformadores de la clásica lógica aristotélica en la lógica de primer orden. Boole desarrolló el álgebra booleana sobre la que se asienta la computación actual y, en consecuencia, la electrónica digital. De Morgan ideó cómo simplificar por procedimientos matemáticos 
problemas lógicos que solo admiten estados de verdadero o falso. Las aportaciones de Luigi Menabrea, considerado el primero en publicar un artículo científico en la historia de la informática, sobre la calculadora gigante de Charles Babbage (17911871) sirvieron a Ada Lovelace (1815-1852) para realizar la primera programación informática de la historia, aunque la calculadora que Babbage había teorizado acabo siendo construida por Torres Quevedo (1852-1936), autor también del primer videojuego de la historia, El Ajedrecista. Torres Quevedo provocó el salto de máquinas analógicas a electromecanicas al desarrollar

"su primer computador, bautizado como 'el aritmómetro', una máquina electromecánica capaz de realizar cálculos de forma automática con un dispositivo de entrada de comandos -una máquina de escribir-, una unidad de procesamiento -un sistema de poleas, agujas, escobillas, electroimanes y conmutadores- y un dispositivo de salida -de nuevo, una máquina de escribir-" (Belda: 2019, p. 31).

Las máquinas realizaban tareas programadas, pero ya se alzaban voces que adelantaban que en el futuro podría ejecutar tareas para las que fuera necesario pensar.

Solo algunas monografías recorren el camino deteniéndose en destacar aportaciones significativas de Isidoro de Sevilla (556-636) pionero en la compilación que sentó los principios de las bases de datos que con el tiempo se organizaron con estructuras complejas en su evolución de jerárquicas a relacionales y a sistemas de gestión NoSQL para big data: también es reseñable el aporte del matemático persa Al-Juarism (780-850), cuya obra se toma como punto de partida del álgebra y su sucesión de instrucciones concretas y ordenadas como punto de arranque de los algoritmos; o Ramón Llul, desde 2001 patrono de la ingeniería informática, entre cuyas contribuciones está el Ars Magna una máquina capaz de realiza demostraciones lógicas.

"La palabra algoritmo proviene del nombre del matemático persa del siglo VII Muhammad ibn Musa al-Khwarizmi. Cuando posteriormente en el siglo XII se tradujo al latín su libro sobre álgebra: Algoritmo de Numero Indorum pasó él mismo a conocerse como Algoritmi o Al-Juarismi. También deriva de su nombre el término guarismo y a partir del título de otro de sus libros "al-jabr w'al-muqabala" se acuñó el término álgebra (Gandz, 1926)”, (Seijo:2020, p.44).

Las predicciones de Alan Turing (1912-1954) sobre la inteligencia de las máquinas y su propuesta de test, metafórico, para determinar si un interlocutor es capaz de adivinar a través de preguntas y respuestas si habla con una máquina o con humano, propuesto por Turing en 1950, como el juego de imitación (Imitation Game). Considerado un ejemplo probatorio de que las máquinas apostaban a actuar como humanos, tuvo una sonora respuesta de Searle (1980) quien contraponía, a través de su Chinese Room, que la máquina solo simula comprender pero que carece de esa facultad.

En 1956, poco después de la muerte de Turing, se acuña el término inteligencia artificial en la conferencia de Darmouth, un encuentro programado por el informático Jhon McCarthy para reunir durante dos meses a diez mentes prodigiosas "para proceder sobre la base de la conjetura de que cada aspecto del aprendizaje o cualquier otra característica de la inteligencia puede, en principio, ser descrito con tanta precisión que puede fabricarse una máquina para simularlo" (2019, p. 58) De esa 
reunión surgen también los dos modos de entender la IA que han venido pugnando entre sí, desde entonces: bottom-up, o modelo conexionista, que defiende que se debe trabajar en generar hardware que imite la biología cerebral creando la neurona artificial y la red neuronal; y top-down, que aboga por crear sistemas artificiales capaces de trabajar mediante sistemas simbólicos para manipular los símbolos con las reglas que lo hace el cerebro.

En comunicación, Los trabajos de Powers (2012); Karlsen \& Stavelin (2014) sobre el impacto de la tecnología y Flew et al., (2012) el uso de ordenadores para promover la interactividad abre un agenda en la que el análisis se va completando con las aportaciones entre otros, Van Dalen (2012); Clerwall (2014); Edge (2014); Latar (2015); Stavelin (2014); Diakopoulos (2014 y 2019); Carlson (2015); Oremus (2015); Lecompte (2015); Dörr (2016); Graefe (2016); Fanta (2017); Hansen et al. (2017; Lindén (2017); Marconi y Siegman (2017); Oppenheimer (2018), Wölker \& Powell (2018), Brossi et al. (2019), Dierickx (2019), Helberger et al. (2019), Saurwein (2019), Slaček y Tomanic' (2019) Soffer (2019), Yanfang (2019) y Gran y Booth, Bucher (2020), Además de los informes periódicos IDC, SASM Intel y Deloitte, la EBU o Reuters y la Universidad de Oxford.

En España, la producción científica sobre el tema es reciente. Destacan las aportaciones de Salazar (2018), Túñez-López, Toural-Bran y Cacheiro-Requeijo (2018), Vállez y Codina (2018), Essenfelder, Canavilhas, Maia y Pinto (2019), Flores (2019), Rojas y Toural (2019), Rojas-Torrijos (2019), Segarra-Saavedra, Cristòfol y Martínez-Sala (2019), Túñez-López, Toural-Bran y Valdiviezo (2019), Ufarte y Manfredi (2019) y Valdiviezo-Abad y Bonini (2019), Ufarte Ruiz, Fieiras-Ceide, Túñez-López (2020), Túñez-López, Toural-Bran \& Frazão-Nogueira (2020) y Túñez-López, Fieiras-Ceide y Vaz-Álvarez (2021).

Al revisar estos aportes se constata que la comunicación se ha transformado de un proceso lineal de uno a muchos a una relación sistémica de todos con todos en el que el discurso global se va modificando a medida que se difunde por la interacción de los usuarios organizados en redes de distribución. Los públicos tienen más autonomía, pero sus actuaciones dejan un rastro que, a gran escala, permite dibujar patrones de actuación que orienta de modo silente ese comportamiento y, por tanto, aunque no de un modo consciente para el usuario, lo limitan o, al menos, lo condicional.

Las visiones menos complacientes dibujan la IA como un nuevo modo de organizar y controlar las relaciones comerciales generando un nuevo control capitalista virtual, o como un sistema diseñado para generar esferas de control social a través de productos o mensajes singularizados ajustados a necesidades detectadas por el procesamiento de datos masivos. El interés del control social se desplaza a las pautas para crear y organizar las bases de datos y a los sesgos que se incorporen en la creación del algoritmo que las interprete y defina comportamientos. No es el objeto de este estudio profundizar en estos debates sino sistematizar la producción científica más reciente en torno al impacto sobre la Comunicación para identificar tendencias.

El futuro ofrece un abanico casi ilimitado de posibilidades de impacto, "pero no específico de la comunicación ni del periodismo sino de la transformación social: reconocimiento de emociones en le manejo de relaciones, microsegmentación de las audiencias para diseñar/ofertar productos de modo individualizado, posibilidad de crear productos bajo demanda de modo inmediato y personalizado, o disponibilidad instantánea de contenidos en multilenguaje y ajustada a cualquier idioma, incluso con 
ajustes programados para ser difundida con diferentes tipos de vOz o presentada con distintos prototipos de personas" (Túñez-López, Fieiras-Ceide y Vaz-Álvarez: 2021, 190).

\section{Metodología}

Esta investigación se diseñó como análisis cualitativo de datos para poder realizar una evaluación interpretativa de textos a partir de preguntas abiertas para las que se busca una respuesta no predeterminada. Se descarta el estudio descriptivo porque no se persigue la identificación de propuestas sino, a partir de ellas, la identificación de tendencias, por lo que la investigación se aproxima a técnicas interpretativas. En línea con las propuestas de Huberman y Miles, se pretende la selección y codificación de datos, su análisis reflexivo y estructurado para llegar a conclusiones que se orienten a la identificación de patrones y tendencias. Se persigue la comprensión en profundidad del fenómeno de estudio que pudiera identificarse con las tres fases de investigación cualitativa que refieren Taylor-Bogdan (1984).

Se diseña, por tanto, una investigación documental cualitativa que centra su interés en la producción científica sobre el impacto de la tecnología en la gestión de la comunicación, de un modo global, y de la identificación de cambios y tendencias por la aplicación de la IA, de modo más concreto. Es una investigación de revisión narrativa sin resultados concluyentes sino con aportaciones que ayuden a comprender el estado de la cuestión sobre este problema de investigación concreto. No debería contemplarse como un planteamiento de inducción analítica porque no se pretende verificar la validez de teorías o aportaciones sino interrelacionarlas para extraer nuevas propuestas.

Al definir el método de recogida de datos se diseña una consulta a bases de datos representativas de la producción científica en el área de Comunicación. Tomando en consideración que, como señalan Ortuña-Malea, Martín-Martín, Ayllón y López-Cózar, "las bases de datos clásicas, pese a tener una cobertura notable, constituyen sistemas cerrados basados en la selección controlada de una colección de revistas" (2016, p. 129), se centra la búsqueda bibliográfica en Google Scholar (GS). Harzing (2016, p. 16) sostiene que GS "ha recorrido un largo camino desde sus primeros días. Su cobertura ha mejorado de forma espectacular y en la actualidad es mejor que Web of Science o Scopus para cualquier disciplina (Harzing y Alakansas, 2016)".

Orduña-Malea, Martín-Martín, Ayllón y López-Cózar sostienen que se puede "considerar a Google Scholar como un sistema de big data académico" (2016, 1299 y lo definen como "la mayor base de datos académica que existe en la actualidad: un producto que incluye todas las tipologías documentales en la mayoría de idiomas y con una tasa de crecimiento superior tanto a Web of Science Core Collection como a Scopus" (2016, p. 128) en aspectos como la citación, que no es objeto de esta investigación, ha puesto "luz donde antes no había más que sombra, especialmente en las Ciencias Sociales y Humanidades" (2016, p. 130). La búsqueda se repite en WoS y en Scopus.

Se trabaja con combinaciones, a modo de cadenas de palabras de búsqueda, como comunicación e inteligencia artificial, comunicación y robotización, comunicación y algoritmos, comunicación y tendencias, comunicación y tecnología. Los resultados se refinan para eliminar aportaciones duplicadas y otras que no sean artículos publicados en revistas científicas o monografías académicas. La selección se filtra a 
través de la lectura del abstract para reducir la muestra a textos que realmente inciden sobre impacto tecnológico en ámbito de gestión de comunicación. Se obtiene una muestra final de análisis que, por el sesgo selectivo, debe considerarse intencional, de 86 documentos con desarrollo longitudinal centrado en los últimos cinco años (2016 a 2020) y considerando cada propuesta individual como parte de una autoría múltiple-colectiva que se convierte en el auténtico sujeto de análisis. Se acota en el último quinquenio porque el impacto de la IA en la comunicación es un asunto de reciente interés y porque se pretende identificar cambios y transformaciones que orienten al investigador hacia la interpretación de las aportaciones como base para definir tendencias que se proyecten en el tiempo. Se identifican los documentos que han incidido en definir el impacto tecnológico en la comunicación, especialmente los que se derivan de aplicar inteligencia artificial.

No hay, por tanto, hipótesis de partida sino preguntas que, a modo de objetivos de investigación, ayuden a un análisis de las aportaciones que se analizan: ¿cuáles son los temas recurrentes en las publicaciones?, ¿cuáles son los campos de mayor impacto?, ¿cuáles son las principales transformaciones se han producido?, ¿qué previsiones de evolución se pueden identificar? El problema de investigación que se plantea es triple: identificar, a partir, de la interpretación, la incidencia de ese impacto; señalar asuntos de interés para comunicadores e investigadores del área; y definir algunas tendencias de evolución futura de ese impacto, a modo de diagnóstico interpretativo del estado de la cuestión.

\section{Resultados}

El modo en que se están transformando las relaciones online y offline produce una hibridación entre ambos escenarios por el continuo trasvase de acciones entre uno y otro.

"Internet ha abierto un universo infinito de posibilidades de intercomunicación, pero a la vez se ha convertido en una tela de araña en la que caemos atrapados con limitación de movimientos. Es una constante: cada día nos abrimos más a entornos online para ejercitar relaciones offline desde terminales de movilidad. Cada vez nos creemos más libres, más autónomos. A medida que esa sensación crece, aumenta nuestra dependencia del soporte y de la conexión". (Costa-Sánchez, Túñez-López, Míguez-González, 2020).

Esta hibridación queda patente en el análisis cualitativo de la revisión bibliográfica, que permite identificar los temas recurrentes y seleccionar como definitorias las aportaciones singulares que caracterizan la relación IA-Comunicación. Entre ellos se identifican:

\subsection{La semántica.}

Hay un uso, a veces equívoco, de conceptos que, pese a sus diferencias, se utilizan recurrentemente, como definidores genéricos y (falsamente) sinónimos: robotización y automatización; robot o bot y algoritmo; inteligencia artificial y desarrollo tecnológico. También expresiones que denotan un esfuerzo de síntesis, como informática cognitiva o marketing inteligente. 
La informática cognitiva recurre a algoritmos de autoaprendizaje para que el ordenador replique los mismos procesos que seguiría un humano. Se trata de logar que el sistema informativo sea capaz de ser programado para que imite a un cerebro humano en sus tomas de decisiones y sus respuestas. La identificación de patrones y el procesamiento de lenguaje natural a través de la aplicación de minería de datos son las claves.

Aplicado a la comunicación, la etiqueta más descriptiva es la de marketing inteligente, una expresión usada por Lies (2019) al desarrollar la aplicación de lo que Liao et al. (2015) conceptuó como informática cognitiva al analizar la inteligencia artificial aplicada para extraer conocimiento de las acciones de marketing en redes sociales y en webs de compra online. Les explica cómo las técnicas del marketing digital se están convirtiendo en técnicas de ingeniería social en marketing, es decir en lo que sería una interacción de "marketing intelligence and big data".

\subsection{Gobernanza.}

Se asocia al proceder y se vincula en muchas ocasiones a la necesidad de fijar normas referenciales y el rango de las mismas. El informe de "Perrault (2019) es una buena fotografía del estado de la cuestión sobre el interés en preparar medidas para la gobernanza de la Inteligencia Artificial porque señala que solo 19 países miembros de Naciones Unidas disponen realmente de un plan.

"La gobernanza de la IA constituye, posiblemente, uno de los mayores desafíos que se ha planteado a la ciencia y a la técnica jurídicas. [...] Funcionalmente, la IA no es una categoría estanca, sino permeable, transversal, porque actúa sobre los elementos y realidades preexistentes afectando e interfiriendo en su desarrollo y funcionamiento. No es fácilmente comprensible desde modelos convencionales de pensamiento, ni es posible proceder a su gestión y regulación exclusivamente desde postulados tradicionales, previos, creados para una sociedad en la que no existía un desarrollo científico y tecnológico con ese alcance, contenido y naturaleza" (Robles, 2020, p. 20).

Martínez y Vega insisten en la importancia de contar con un modelo de gobernanza inclusivo, perdurable y dinámico, en los principios éticos y en la necesidad de crear capital humano. Sobre gobernanza e AI destacan cinco aspectos:

"Primero, se requiere la cooperación de todos los sectores tanto en el diseño como en la "implementación", evaluación, y constante actualización de la PNIA. Segundo, se necesita que los legisladores y los diseñadores de políticas públicas se comprometan con el uso ético de la IA. Tercero, el proceso de gobernanza de la IA debe estar descentralizado en todos los sectores de la administración pública y evitar que la innovación se concentre en un solo ministerio. Cuarto, deben respetarse los derechos de las personas en el uso de la tecnología, tales como el principio de una sola vez; el acceso a internet; acceso a la información; la protección de datos personales en internet. Quinto, es importante considerar el marco de referencia internacional sobre estándares éticos de tecnologías emergentes como la IA, pero también atender las características culturales, jurídicas y de nivel de desarrollo de cada país" (2020: p. 16). 
Globalmente, en el entorno europeo, el panorama se puede observar a través de la definición de IA como expresión que se aplica a los sistemas que manifiestan un comportamiento inteligente, pues son capaces de analizar su entorno y pasar a la acción - con cierto grado de autonomía - con el fin de alcanzar objetivos específicos (COM (2018) 237, p. 1) recogida en la 'Declaración de cooperación en Inteligencia Artificial (IA)' que la UE hizo pública en 2018, como paso previo al libro blanco sobre IA que publicó en 2020.

En España, se espera el desarrollo de acciones para ajustarse al plan europeo. Hasta la fecha, la referencia es el documento sobre I+D+i en IA con el que, en 2019, el Gobierno asume que la IA será un "eje estratégico de la sociedad española del siglo XXI" que supondrá una "revolución tecnológica y social" y que afectará "el mercado laboral, el modelo educativo, la legislación en vigor y las relaciones dentro de la propia sociedad con los nuevos servicios y sistemas desarrollados" (2019, p. 9). En ese texto se señalan cuales van a ser las prioridades: un Observatorio Nacional de IA, establecer áreas estratégicas, intensificar la educación en IA y reforzar la aplicación de IA en la enseñanza.

\subsection{Transversalidad e bibridación de campos.}

Dada su complejidad, el desarrollo de la investigación científica y tecnológica de los sistemas inteligentes requiere del apoyo de otras áreas del conocimiento, tales como: Filosofía, Psicología, Lingüística, Ciencias de Computación, Biología, Neurociencias, Matemática, Física, Química, Cibernética, Electrónica y Comunicaciones.

"La antropología, la filosofía, la sociología, la política, la economía, las ciencias matemáticas y físicas, las ingenierías y, también, el derecho y la ciencia jurídica, la ética y hasta la moral se encuentran frente a un fenómeno de una magnitud desconocida, inconmensurable, imparable y, por todo ello, inquietante" (Robles, 2014, p. 2).

Paredes y Quinde ejemplarizante bien esa multidisciplinariedad a través de Russell y Norving (1996):

"En filosofía, han surgido teorías de razonamiento y aprendizaje, junto al punto de vista, de que la mente está constituida por la operación de un sistema físico. En matemáticas, aparecen las teorías formales de lógica, toma de decisiones, probabilidad y computación. En psicología, se crean las herramientas para investigar la mente humana. En lingüística, las teorías de la estructura y significado del lenguaje. En informática, se instaura las herramientas para hacer realidad la IA" (2020, p. 18)

\subsection{IoT.}

Interesa todo lo que tiene que ver con las gestión de comunicación y de relaciones a partir de la aplicación de IA en el IoT (Internet of Things o Internet de las cosas), tanto en lo que supone programar para dotar de autonomía a las cosas para que puedan interactuar con otras cosas o con humanos a través de internet como prestar atención al desarrollo posibilidades de interacción al combinar IoT con geolocalización y movilidad a través de terminales en movilidad, no solo por lo que supone de 
puntos de contacto casi permanente con los públicos sino porque representa una fuente constante de suministro de información (Cuervo Sánchez, 2021). Y, también, porque se da un bucle en el uso: cada vez más fácil e intuitivo y cada vez en las ampliaciones que se usan como herramientas de estrategias de marketing o de comunicación (Muñoz-Leiva, Climent-Climent y Liébana-Cabanillas, 2017).

\subsection{Heteromation, cobotización, robotlución.}

La preocupación por el impacto en trabajo, que algunos autores refieren como beteromation (Ekbia y Nardi, 2017; Dholakia y Firat, 2019). Cuervo Sánchez (2021, p. 38) destaca que "en los últimos años, el interés por la IA y su impacto en el marketing y, en particular en aquel de bienes de consumo, ha vuelto a cobrar impulso" y recurre a Grandinetti (2020) para explicar que se habla de cambios radicales que ya, como ya explicaban Kotane, Znotina y Hushko (2019), no se ciñen únicamente a la comercialización de productos, sino que se amplía cada vez más al uso de IA para singularizar la recomendación de productos.

La automatización y la informatización de procesos se ha visto como una amenaza para determinados perfiles profesionales que se centran en tareas que pasan a ser íntegramente realizadas por máquinas o por programas informáticos. En la cara opuesta, la IA genera nuevas oportunidades laborales para atender nuevas demandas de actuación generadas a partir de la implantación de las transformaciones que provoca. En ámbitos laborales, en la dicotomía sobre la IA como amenaza o refuerzo de actividad laboral y su repercusión sobre los índices de empleo se cuela de la convergencia de nuevos "trabajadores digitales" que en ocasiones provocan la demanda de recalificación profesional y un entorno laboral en el que máquinas inteligentes o robots comparten labores y tareas con humanos. Este coworking entre personas y máquinas se ha empezado a referir como "cobotización" (Béliz, 2018, en Corvalán, 2019).

"La receta de la cobotización para evitar la pérdida de empleo es atizar la creación de una fuerza laboral con inteligencia aumentada, donde la IA eleve los límites de las capacidades tradicionales. Con el surgimiento de los "trabajadores digitales", casi tres cuartos del impacto de la automatización en el empleo se producirá dentro del mismo puesto de trabajo", (Bellocchio, 2019).

La cobotización no es exclusiva del sector de la comunicación, aunque la informatización de todo el proceso de generación de contenidos pudiera hacer pensar esa inevitable convergencia entre trabajadores/as humanos/as y trabajadores/as digitales ya que, como señala en Robotlución, una obra colectiva impulsada por el Banco Interamericano de Desarrollo en su informe (2017) (https://urlzs.com/i2vf9), la cobotización inclusiva se orienta sectores que son clave en el manejo de todas las actividades humanas: asistencia inteligente; automatización; diagnóstico inteligente; y predicción”.

\subsection{Gig economy y orange economy.}

Mientras el debate se orienta a la permanencia o destrucción de índices de empleo, se van abriendo paso las voces que advierten de un cambio en el modelo productivo y en el efecto de la IA sobre los modos de vinculación contractual con las empresas. 
Al discurso de la innovación y la actualización constante se une el hilo, cada vez más frecuente, sobre el emprendimiento, los perfiles personales como identificación laboral y el trabajo freelance en un panorama que dibuja los elementos de la gig economy: un término inspirado en las actuaciones musicales (los populares bolos) para definir tareas o vinculaciones de corta duración, vinculadas a al proyecto a la vigencia de la solución que se implementa, y que Iglesias (2018) define como "consiste en aceptar encargos de una duración concreta y sin exclusividad con la empresa contratante"

El impacto en empresas, productos servicios y manejo de relaciones en comunicación se vincula a actividades de lo que se ha dado en etiquetar como orange economy (economía naranja), en la que se engloban las actividades que transforman el conocimiento en un bien que persigue a la vez beneficio económico y el desarrollo cultural. El concepto de economía naranja se vincula a las industrias creativas y particularmente

"a dos grandes áreas. Por un lado, los bienes y servicios que se generan en el arte, el diseño, la música, la moda, la artesanía y diversas formas de entretenimiento. Por otra parte, las plataformas y los sistemas de innovación que dan soporte a la creatividad asociada a la estética, al aspecto de los bienes y servicios y en los cambios emocionales que esos productos generan en los consumidores" (Corvalán, 2019, p. 43).

\subsection{Adprosumers.}

Se señala un movimiento cíclico en una espiral de participación y personalización. Se identifican voces que inciden en que "si bien el papel del mercado en la innovación es importante, la regulación de la inteligencia artificial en el marco de la Unión Europea debe tener en cuenta a las personas" (Sánchez Bravo, 2020, p. 65). La IA no está solo en grandes aplicaciones. A veces pasa inadvertida, aunque esté presente en acciones cotidianas como los captchas, surgidos en el 2000 de una investigación académica en la universidad Carnegie Mellon (Pittsburgh) por el grupo de investigación liderado por el guatemalteco Luis von Ahn. Captcha remite al test de Turing porque significa Completely Automated Public Turing test y se usa para probar que al otro lado no hay un algoritmo sino un humano (Seijo, 2020).

Los consumidores han evolucionado a prosumidores (productores y consumidores). Y de ahí a adprosumidores: consumen, producen y evalúan. Surgen nuevos roles a la vez que aumentan las huellas y el rastro digital y aumenta el caudal de información que dejamos en la red y, en base a esos datos, se incrementan las soluciones singularizadas.

"Para inferir resultados con menor margen de error la IA necesita de una gran cantidad de datos. A mayor capacidad de información, especialmente en la fase de entrenamiento del algoritmo, mayor fiabilidad se desprenderá del análisis de la IA. Y, desde la perspectiva opuesta, a mayor cantidad de datos suministrados, mayor probabilidad habrá de que se transgredan ciertas líneas en cuanto a la privacidad y suministro de información personal de los ciudadanos" (Castellanos, 2020, p. 71).

\subsection{Derecho y ética.}

Los discursos se bifurcan entre los estudios que analizan los avances y las consecuencias de aplicar IA en ámbitos del derecho y los que debaten el rol de aplicación 
de la IA al derecho y las aportaciones en torno a la regulación de la IA desde el derecho. Simplificando, la discusión es triangular porque mientras se alzan voces de los que ven en las normas o en las propuestas de legislar un freno a la transformación tecnológica, se mantienen las diferencias entre los que defienden que la regulación se puede dar simplemente con códigos de ética y los que consideran que se debe proceder para superar el vacío jurídico porque apelar a principios deontológicos y éticos como modo de resolver las necesidades doctrinales es una forma de cuestionar la propia función del derecho.

El uso de la inteligencia artificial para creaciones artísticas o para resultados creativos abre otra vía de discusión sobre la protección de la obra algorítmica con atribución al derecho de autor (Saiz García, 2019) y sobre el modo de atribuir el derecho de autor de una obra algorítmica. "Ante una ausencia absoluta de actividad intelectual humana nos preguntamos cuál debería ser la correcta solución, si su protección por un derecho conexo o sui generis o, en definitiva, si no es necesario crear ninguna categoría especial" (2019, 1).

Es un enfoque de interés toda vez que la IA ha irrumpido, también, en la generación de productos culturales: música, pintura, poesía, cortos, etc. Miranda (2020) apunta que

"el artista es la mente maestra detrás de la obra de arte" independientemente de si la tarea la ejecutó un humano o una máquina. En su opinión "el crédito se debe dar al individuo (o a los colaboradores) detrás de la obra" (2019). Sus propuestas se ilustran con ejemplos como el caso del británico Harold Cohen, "que creó cientos de imágenes (autorretratos) a partir de una computadora" y el proyecto Criptobloom que "plantea obras como Myriad (Tulips), un conjunto de entrenamiento para alimentar una base de datos con diez mil imágenes de tulipanes" (2019).

\subsection{GAFAM.}

Cobertura, publicidad, infraestructura, recopilación de datos...se concentran en cinco grandes nombres: Google, Amazon, Facebook, Apple y Microsoft, conocidas en conjunto a través del acrónimo formado por sus iniciales: GAFAM que, como señalan Miguel De Bustos e Izquierdo-Castillo (2019),

"reúnen condiciones necesarias para ser considerados los líderes de la economía actual, caracterizada también como segunda economía (Arthur, 2011), o capitalismo de vigilancia (Zuboff, 2016). Esto condiciona al resto de industrias vinculadas de forma competitiva con ellos, como son las industrias culturales".

Se presentan a modo de círculo concéntrico, con más posibilidad de desarrollo tecnológico por mayor capacidad económica y de aumento de la capacidad económica por la aplicación de soluciones que le permiten diferenciarse de los competidores y hacer con posiciones de dominio. "A través de la innovación, los GAFAM generan grandes barreras de entrada en el macro ecosistema que forman. Aun así, las plataformas mediáticas disruptoras permanecen líderes en sus respectivos sectores, donde los GAFAM encuentran resistencia" (Miguel De Bustos e Izquierdo-Castillo, 2019), entre los que refieren: Netflix en el VOD (video on demand) en competencia 
con Amazon Prime Video, Disney y HBO; o Spotify en la música en streaming frente a las propuestas de otros gigantes como Google, Amazon o Apple.

Es importante el apunte porque limitan la capacidad de acción y constriñen la capacidad de generación de nuevas industrias culturales y la circulación de productos y propuestas de comunicación en un cuestionable pulso a la universalización de la cultura y el conocimiento. De Bustos e Izquierdo-Castillo advierten que

"debemos considerar que en la medida en que los GAFAM incorporan las áreas creativas y culturales a sus ecosistemas, la capacidad de competencia desde agentes externos se debilita. Incluso en el supuesto que una empresa externa consolide posiciones en alguno de los sectores, los GAFAM buscarán anexionarla y, en el caso de no ser viable, competir directamente para desbancarla" (2019).

\subsection{Ludificación.}

IA en ludificación y ludificación como escenario comunicativo y de interacción con la audiencia. En cierto modo, la programación algorítmica es la base de la ludificación, que crece como escenario de interacción con usuarios y de penetración en targets específicos, incluso en actuaciones de índole político desvinculadas de lo que son actividades o temáticas propias de ese entorno. Se recurre a la IA para que el juego tenga autonomía en la toma de decisiones que permita variabilidad de acciones. La IA se usa "para aumentar la interacción y evitar la repetitividad del juego creando motores gráficos que permiten a los personajes actuar según su contexto y en respuesta a las ejecuciones del jugador" (Bernal, Cardona y Páez, 2018, p. 17)

\subsection{Gestión de la comunicación.}

Al revisar la literatura sobre efectos de la IA en el marketing, Cuervo Sánchez (2021, p. 33) destaca que

"el uso de la IA ha sido más evidente en el caso de empresas que están dirigidas al consumidor final (B2C). Las tecnologías de la IA aplicadas al marketing transforman la manera de interactuar con clientes; es así como, surge un ecosistema enfocado en diseñar estrategias competitivas con facultades para predecir comportamientos. Finalmente, con la aplicación de las tecnologías de la IA al marketing, surge una nueva experiencia del consumidor por la personalización del servicio, y las actividades creativas del equipo de marketing adquieren relevancia porque potencian las innovaciones en diseño de productos ajustados a los intereses del consumidor" (Medina-Chicaiza y Martínez-Ortega, 2020, p. 44).

La hipersegmentación de audiencias y su microconocimiento impulsan el crecimiento de dinámicas comunicativas al mismo tiempo que cambios de actitudes sociales. Un ejemplo con los washing, como el greenwashing, vinculado a ecología como motor de consumo, o el pinkwashing que se orientan a ámbitos reivindicativos femeninos o de concienciación y lucha social como el cáncer de mama. 
Al concretar el análisis en comunicación, queda patente que al referir la potencialidad e impacto de la IA hablamos de productos de todo tipo elaborados en multilenguaje: textual, visual y sonoro. Y, también, que el uso depende de la intencionalidad de las personas que la apliquen porque una misma posibilidad puede tener finalidades positivas o servir para desarrollar agresiones físicas y psicológicas. En los usos perversos, el área que parece prioritaria es la identificación de elementos de ficción que se presentan como relatos de lo verídico para simular ser noticias que transmitan contenidos falsos. La IA es de doble filo porque los expertos reconocen que a la vez que se trabaja para detectar las fakes (falsos por imitación visual -deepfake-, de voz o de relatos no verídicos elaborados con convenciones periodísticas -noticias-, por ejemplo) se trabaja en programar el modo de eludir esos reconocimientos, es decir de blindaje de la falsa noticia ante los verificadores.

Medina-Chicaiza y Martínez-Ortega, que también revisan la literatura sobre IA y Marketing, concluyen que

"de las múltiples tecnologías de la IA existentes en la literatura científica, la inclusión de la IA en la empresa es posible con la implementación de: Aprendizaje automático (machine learning), procesamiento del lenguaje natural (PLN), reconocimiento visual (visual recognition), reconocimiento de texto (text recognition), big data, aprendizaje profundo (deep learning), nube (cloud computing), internet de las cosas (IoT) en los procesos que integran la cadena de valor empresarial" (2020, p. 44).

Hay coincidencia en señalar como áreas de impacto las que siguen:

- Big Data. Recogida, almacenamiento y tratamiento de ingentes cantidades de datos que se transformen en indicaciones para la toma de decisiones.

- Machine Learning, o aprendizaje automático, que se refiere a que las programaciones incluyen la posibilidad de corregir errores en base a la experiencia de ejecución de la acción programada. Puede ser un aprendizaje dirigido (Supervised Learning) para el que se entrena a la máquina en encontrar soluciones analizando datos etiquetados para que proceda del mismo modo con datos nuevos. Son la base de los recomendadores de contenidos porque se basan en acciones previas para singularizar la acción y aproximarla a mensajes personalizados. También puede ser un aprendizaje no dirigido (Unsupervised Learning) si no incluyen etiquetas, que se corresponde con acciones de aprendizaje por refuerzo o para diseñar acciones de publicidad programática. Machine Learning se aplica en todo tipo de decisiones, tanto en la optimización de precios como en refinar los motores de búsqueda o en servir de base para la segmentación de públicos.

- Deep Learning, o un programa algorítmico aprendizaje automático que predice acciones futuras basándose en modelos que ya existen. Objetos autónomos, como los coches, son un ejemplo, pero también análisis predictivos de riesgo o de evaluación de tratamientos médicos. Es importante en comunicación, por su triple vertiente: de trabajo con imágenes faciales (además de aplicarse en sistemas de seguridad por reconocimiento), de respuesta oral o escrita a mensajes enviados por lenguaje natural y por su aplicación en la visión computacional, "permite un mejor entendimiento en la interpretación de imágenes, mejor que los métodos tradicionales" (Paredes y Quinde (2020)). 
Otro campo de preocupación es la educación y el aprendizaje en IA y para IA (alfabetización, más allá de las habilidades digitales). El análisis de Ufarte Ruiz; Fieiras-Ceide y Túñez-López, revisa 768 asignaturas de 17 planes de estudios de grado y 116 materias de 8 másteres oficiales y concluye que la oferta educativa resulta insuficiente porque no hay propuestas específicas que inciden las de roles, rutinas y convenciones derivadas del impacto de la tecnología y su "revolución transformadora", que incluso "en la relación con el estudiantado derivará hacia una enseñanza ultrapersonalizada tanto en entornos presenciales como en docencia en línea” (2020, p. 133). En sus conclusiones señalan que las asignaturas que se orienten a la robotización y la automatización "son inexistentes "en los estudios de grado de Comunicación, aunque "se atisban algunas propuestas" en los estudios de máster.

"Esta carencia subraya la importancia de generar materias especializadas en la oferta curricular y es un ejemplo más de cómo las TIC, en continua evolución, y sus disciplinas afines van más deprisa que el mundo educativo" (2020, p. 142).

\section{Conclusiones}

La revisión bibliográfica evidencia que están cambiando los roles porque se transforman los escenarios y las funciones, con nuevas actitudes y nuevos conceptos. La hibridación de soportes se traslada a la hibridación de perfiles y de necesidades (cobotización). La gobernanza de la IA está en construcción, al igual que las aportaciones del sector y su uso en comunicación, como el marco de impulso gubernamental en el Plan estratégico pendiente de aprobación.

Los cambios se hacen visibles en la medida en la que afectan al comportamiento de los públicos, pero de modo silente ya han transformado del modo de comunicar: de elaborar, de envasar, de distribuir y de consumir productos y contenidos. Los ajustes en las rutinas y en los perfiles provocan restricciones de necesidades y funciones a la vez que demandan una nueva actitud: menos operatividad y más estrategia, menos discursos y más interacción, menos elaboración cerrada y más participación en la construcción de contenidos, menos reiteración y más innovación, menos comportamientos rutinarios y más aporte cognitivo en la ideación de soportes y contenidos.

En esta revisión bibliográfica cumple su propósito de identificar los ítems que definen como la revolución tecnológica está afectando a la comunicación y se propone casi una treintena de apuntes que definen en trazo grueso las tendencias del impacto de la inteligencia artificial en la comunicación y en su gestión en un contexto en el que se da un cambio permanente porque hay una constante que altera sus procesos e incluso varía el rol que asumen las organizaciones que se ven empujadas a comportarse en entornos online como un actor más que se relaciona de modo directo con los otros actores que integran sus públicos y pasan de comunicar para sus públicos a comunicar siendo público. 


\section{Bibliografía}

Belda, I. (2019). Inteligencia Artificial. De los circuitos a las máquinas pensantes. Barcelona:RBA

Bellocchio, L. (2019) ¿Qué es la cobotización inclusiva?, en https://valor.pe/que-es-la-cobotizacion -inclusiva/

Bernal Rozo, E. Cardona Montoya, R., \& Páez, J. (2018). Videojuegos: Avances tecnológicos en aplicación de física e inteligencia artificial. Letras ConCiencia TecnoLógica, 61-78. En https://revistas.itc.edu.co/index.php/letras/article/view/114

Brossi, L., Dodds, T. y Passeron, E. (2019). Inteligencia artificial y bienestar de las juventudes en América Latina. 1ra Edición, LOM Ediciones, Chile. pp. 5-6.

Carlson, M. (2015). The robotic reporter: automated journalism and the redefinition of labor, compositional forms, and journalistic authority. Digital journalism 3(3), pp. 416-431.

Carr, N. (2011). Superficiales: Qué está haciendo internet con el impacto cerebral de Internet nues-tras mentes. Madrid: Taurus. 3

Castellanos, J. (2020). La gestión de la información en el paradigma algorítmico: inteligencia artificial y protección de datos. Métodos de Información, 11(21), 59-82 DOI: https://dx.doi. org/10.5557/IIMEI11-N21-059082

COM (2018) 237 Inteligencia artificial para Europa. Comunicación de la Comisión al Parlamento Europeo, al Consejo Europeo, al Consejo, al Comité Económico y Social europeo y al Comité de las Regiones. Recuperado de https://ec.europa.eu/transparency/regdoc/ rep/1/2018/ES/COM-2018-237-F1-ES-MAIN-PART-1.PDF

Corvalán, J.G. (2019). El impacto de la inteligencia artificial en el trabajo. Revista de Direito Econômico e Socioambiental, Curitiba, v. 10, n. 1, pp. 35-51, jan/abr 2019. Doi: 10.7213/ rev.dir.econ.soc.v10i1.2587

Costa-Sánchez, C., Túñez-López, M., \&. Míguez-González, M-I. (2020) Normal is not normative: Public relations indicators from a Spanish secondary political and economic region. Public Rela-tions Inquiry. December 2020. doi:10.1177/2046147X20979290

Cuervo Sánchez, C. A. (2021) Efectos de la inteligencia artificial en las estrategias de marketing: Revisión de literatura. Revista Internacional de Investigación en Comunicación aDResearch ESIC. No 24 Vol 24 Primer semestre, enero-junio 2021 · pp. 26 a 41 https://doi.org/10.7263/ adresic-024-02

Dholakia, N., \& Firat, A. F. (2019). Markets, consumers and society in the age of heteromation. European Journal of Marketing, 53(8), pp. 1504-1520.

Diakopoulos, N. (2014). Algorithmic accountability. Digital Journalism 3(3), pp. 398-415. https:// doi.org/10.1080/21670811.2014.976411

Diakopoulos, N. (2019). Automating the News. London: Harvard University Press

Dierickx, L. (2019). Information automatisée et nouveaux acteurs des processus journalistiques. Sur le journalisme Vol. 8 (2)

Dörr, K. (2016). Mapping the field of algorithmic journalism. Digital Journalism 4(6), pp. 700-722. https://doi.org/10.1080/21670811.2015.1096748

Edge,A.(2014). Ophan:keymetricsinformingeditorialatTheGuardian.Recuperadodehttps://www. journalism.co.uk/news/how-ophan-offers-bespoke-data-to-inform-content-at-the-guardian/ s2/a563349

Ekbia, H., \& Nardi, B. (2017) Heteromation, and Other Stories of Computing and Capitalism. The MIT Press https://doi.org/10.7551/mitpress/10767.001.0001

Essenfelder, R.; Canavilhas, J.; Maia, H. C. y Pinto, R. J. (2019). Automatización de textos periodísticos en la televisión brasileña: Estudio de caso del sistema AIDA (Globo-Brasil). Doxa Comunicación, 29, pp. 255-274.

Fanta, Alexander (2017). Putting Europe's robots on the map: automated journalism in news agencies". Retrieved from: https://reutersinstitute.politics.ox.ac.uk/sites/default/files/2017-09/ Fanta\%2C\%20Putting\%20Europe\%E2\%80\%99s\%20Robots\%20on\%20the\%20Map.pdf 
Flew, T., Spurgeon, CH. \& Swift A (2012). The promise of computational journalism. Journalism Practice 6(2), pp. 157-171. https://doi.org/10.1080/17512786.2011.616655

Flores Vivar, J. M. (2019). Inteligencia artificial y periodismo: diluyendo el impacto de la desinformación y las noticias falsas a través de los bots. Doxa Comunicación, 29, pp. 197-212. https://doi.org/10.31921/doxacom.n29a10

Graefe, A. (2016). Guide to automated journalism. Recuperado de https://www.cjr.org/tow_ center_reports/guide_to_automated_journalism.php

Gran, A.B.; Booth, P. \& Bucher, T. (2020). To be or not to be algorithm aware: a question of a new digital divide? Information, Communication \& Society. 1-18. 10.1080/1369118X.2020. 1736124 .

Grandinetti, R. (2020). How artificial intelligence can change the core of marketing theory. Innovative Marketing, 16(2), pp. 91-103.

Hansen, M., Roca-Sales, M., Keegan, J. et al. (2017) Artificial Intelligence: Practice and Implications for Journalism. Brown Institute for media innovation and the tow center for digital journalism. Columbia Journalism School. https://doi.org/10.7916/d8x92prd

Harzing A.-W. (2016). La revolución Google Scholar. Destapando la caja de Pandora académica. Granada: Universidad de Granada y Unión de Editoriales Universitarias de España (UNE), 2016, 268 págs. ISBN: 978-84-338-5985-3.

Helberger, N., Eskens, S. J., van Drunen, M. Z., Bastian, M. B., \& Möller, J. E. (2019). Implications of AI-driventools in the media for freedom of expression. Paper presented at Artificial intelligence - Intelligent politics:Challenges and opportunities for media and democracy, Nicosia, Cyprus.

Iglesias, M (2018) ¿Qué es la 'gig economy'? BBVA (https://www.bbva.com/es/que-es-la-gig -economy/)

Karlsen, J. \& Stavelin, E. (2014) Computational journalism in norwegian newsrooms. Journalism Practice 8(1):34-48. https://doi.org/10.1080/17512786.2013.813190

Kim, J., Lee, K., Kim, Y., Kuppuswamy, NS. \& Jo, J. (2007). Ubiquitous robot: a new paradigm for integrated services. En: 2007 IEEE international conference on robotics and automation, pp. $2853-2858$

Kotane, I., Znotina, D., \& Hushko, S. (2019). Assessment of trends in application of digital marketing. Periodyk Naukowy Akademii Polonijnej, 33(2), pp. 28-35.

Latar, Noam-Lemelshtrich (2015). "The robot journalist in the age of social physics: the end of human Journalism?" En Einav G. (Eds.) The New World of Transitioned Media: The Economics of Information, Communication, and Entertainment: The Impacts of Digital Technology in the 21st Century (pp. 65-80). Suiza: Springer International Publishing. Lecompte, C. (2015). Automa-tion in the newsroom. Nieman Reports 69(3), pp. 32-45. Recuperado de http://niemanreports.org/wp-content/uploads/2015/08/NRsummer2015.pdf

Lecompte, C. (2015). Automation in the newsroom. Nieman Reports 69(3), pp. 32-45. Recuperado de http://niemanreports.org/wp-content/uploads/2015/08/NRsummer2015.pdf

Liao, S.-H., Hsiao, P.-Y., Li, C.-W., \& Lin, Y.-F. (2015). Mining marketing knowledge to explore social network sites and online purchase behaviors. Applied Artificial Intelligence, 29(7), pp. 697-732.

Lies, J. (2019) Marketing intelligence and big data: Digital marketing techniques on their way to becoming social engineering techniques in marketing. International Journal of Interactive Multimedia \& Artificial Intelligence, 5(5), pp. 134-144.

Lindén, C. (2017). Algorithms for journalism: the future of news work. The Journal of Media Innova-tion 4(1):60-76. https://doi.org/10.5617/jmi.v4i1.2420

Marconi, F. \& Siegman, A. (2017). The future of augmented journalism: a guide for newsrooms in the age of smart machines. About AP insights. Recuperado de https://insights.ap.org/ uploads/images/the-future-of-augmented-journalism_ap-report.pdf

Martínez, Y., \& Vega, M. (2020) Gobernanza participativa de la Inteligencia Artificial. Banco Inter-americano de Desarrollo. En https://publications.iadb.org/publications/spanish/ document/Gobernanza-participativa-de-la-inteligencia-artificial.pdf 
Medina-Chicaiza, P., \& Martínez-Ortega, A. (2020). Tecnologías en la inteligencia artificial para el Marketing: una revisión de la literatura. Pro Sciences, 4(30), pp. 36-47. https://doi. org/10.29018/issn.2588-1000vol4iss30.2020pp36-47

Miguel de Bustos, J.C., \& Izquierdo-Castillo, J. (2019) ¿Quién controlará la Comunicación? El impacto de los GAFAM sobre las industrias mediáticas en el entorno de la economía digital. Revista Latina de Comunicación Social, 2019, núm.74, pp. 803-821 DOI https://doi. org/10.4185/RLCS-2019-1358

Miranda, A. (2020) Inteligencia artificial demasiado humana (aún): arte y tecnología Revista DigitUniversitaria (RDU) Vol. 21, núm. 1 enero-febrero 2020 DOI: http://doi.org/10.22201/ codeic.16076079e.2020.v21n1.a7

Muñoz-Leiva, F., Climent-Climent, S., Liébana-Cabanillas, F. (2017). Determinants of intention to use the mobile ban-king apps: An extension of the classic TAM model. Spanish Journal of Marke-ting-ESIC, 21(1), pp. 25-38.

Oppenheimer, Andrés (2018) Sálvese quien pueda. El futuro del trabajo en la era de la automatización. Madrid: Debate.

Orduña-Malea, E., Martín-Martín, A., Ayllón, J.M., \& Delgado López-Cózar, E. (2016) La revolución Google Scholar. Destapando la caja de Pandora académica. Prólogos de Peter Jacsó y Anne-WilHarzing. Granada: Universidad de Granada y Unión de Editoriales Universitarias de España (UNE), 2016, 268 págs. ISBN: 978-84-338-5985-3.

Oremus, W. (2015). No more pencils, no more books. Slate. Retrieved from http://publicservicesalliance.org/wp-content/uploads/2015/10/Adaptive-learning-software-is-replacing-textbooks-and-upending-American-education.-Should-we-welcome-it.pdf

Paredes C.E., \& Quinde, G.A. (2020) Aplicaciones de la inteligencia artificial en marketing digital. Ambato: Universidad Tecnica Ambato, Ecuador. En http://repositorio.uta.edu.ec/ bitstream/123456789/31487/1/784\%20MKT.pdf

Perrault, R., (2019) The AI Index 2019 Annual Report, AI Index Steering Committee, Human-Centered AI Institute, Stanford University, Stanford, CA, 2019. En https://hai.stanford.edu/ research/ai-index-2019

Powers, Matthew. (2012). "In forms that are familiar and yet-to-be invented: american journalism and the discourse of technologically specific work". Journal of Communication Inquiry 36 (1), 24-43. Robles Carrillo, M. (2014). La gobernanza de la inteligencia artificial: contexto y parámetros generales. Journal of Evolution \& Technology, 24(1), pp. 44-62. Doi:10.17103/ reei.39.07

Robles Carrillo, M. (2014). La gobernanza de la inteligencia artificial: contexto y parámetros generales. Journal of Evolution \& Technology, 24(1), pp. 44-62. Doi:10.17103/reei.39.07

Rojas Torrijos, J. L. \& Toural Bran, C. (2019). Periodismo deportivo automatizado. Estudio de caso de AnaFut, el bot desarrollado por El Confidencial para la escritura de crónicas de fútbol. Doxa Comunicación, 29, pp. 235-254. https://doi.org/10.31921/doxacom.n29a12

Saiz García, C. (2019) Las obras creadas por sistemas de inteligencia artificial y su protección por el derecho de autor (AI Created Works and Their Protection Under Copyright Law) (January 2019). InDret, Vol. 1, 2019, Available at SSRN: https://ssrn.com/abstract=3365458

Salazar, I. (2018). Los robots y la inteligencia artificial. Nuevos retos del periodismo. Doxa Comunicación 27, pp. 295-315. https://doi.org/10.31921/doxacom.n27a15

Sánchez Bravo, A.A. (2020) European framework for people-based artificial intelligence International Journal of Digital Law - IJDL. (1), 1 (abr. 2020)

Saurwein, F. (2019). Emerging structures of control for algorithms on the Internet en Media Ac-countability in the Era of Post-Truth Politics. European Challenges and Perspectives, editado por Eberwein, T., Fengler, S. \& Karmasin, M. (Ed.) Capítulo 13. Abingdon (UK): Routledge.

Searle, J.R., (1980) Minds, brains, and programs, Behavioural and Brain Sciences, $\mathrm{n}^{\circ}$ 3, 1980, pp. $417-457$. 
Segarra-Saavedra, J.; Cristòfol, F. J. \& Martínez-Sala, A. M. (2019). Inteligencia artificial (IA) aplicada a la documentación informativa y redacción periodística deportiva. El caso de BeSoccer. Doxa Comunicación, 29, pp. 275-286. https://doi.org/10.31921/doxacom.n29a14

Seijo, P. (2020) Yo no soy un robot: reflexiones sobre inteligencia artificial y sociedad mediante el ejemplo de los 'captcha'. Tecnología \& Sociedad, Buenos Aires, 9, 2020, pp. 37-54

Slaček, S. \& Tomanić, I. (2019) Algoritmizacija nacionalne tiskovne agencije: primer STA, Javnost - The Public, 26: sup1, S62-S81, DOI: 10.1080 / 13183222.2019.1696601

Soffer, O. (2019). Personalización algorítmica y el flujo de comunicación en dos pasos, Communication Theory, qtz008, https://doi.org/10.1093/ct/qtz008

Stavelin, E. (2014). Computational journalism: when journalism meets programming (Ph.D. dissertation). University of Bergen

Taylor, S.J., \& Bogdan, R. (1984). Introduction to Qualitative Research Methods. The Search for Meanings . New York: Wiley and Sons. (Traducción al español: Introducción a los métodos cualitativos de investigación. Barcelona: Paidós. 1987)

Túñez-López, M., Costa-Sánchez, C., \& Míguez González, M. I. (2018). Avances y retos de la gestión de la comunicación en el siglo XXI. Procesos, necesidades y carencias en el ámbito institucional. Estudios sobre el Mensaje Periodístico, 24(1), pp. 921-940. https://doi. org/10.5209/ESMP.59987

Túñez-López, J. M., Fieiras Ceide, C., \& Vaz-Álvarez, M. (2021). Impact of Artificial Intelligence on Journalism: transformations in the company, products, contents and professional profile. Communication \& Society,34(1), pp. 177-193.

Túñez-López, J.M., Toural-Bran C. \& Frazão-Nogueira A.G. (2020) From Data Journalism to Robotic Journalism: The Automation of News Processing. En Vázquez-Herrero J., Direito-Rebollal S., Silva-Rodríguez A., López-García X. (eds) Journalistic Metamorphosis. Studies in Big Data, vol 70. Springer.

Túñez-López, M., Toural-Bran, C. \& Cacheiro-Requeijo, S. (2018). Uso de bots y algoritmos para automatizar la redacción de noticias: percepción y actitudes de los periodistas en España. El profesional de la información 27(4):750-758. https://doi.org/10.3145/ epi.2018.jul.04

Túñez-Lopez, M., Toural-Bran, C. \& Valdiviezo, C. (2019). Automation, bots and algorithms in newsmaking. Impact and quality of artificial journalism. Revista Latina de Comunicación Social, 74, pp. 1411-1433 http://www.revistalatinacs.org/074paper/1391/74en.html DOI: 10.4185/RLCS-2019-1391en

Ufarte Ruiz, M. J.\& Manfredi Sánchez, J. L. (2019). Algoritmos y bots aplicados al periodismo. El caso de Narrativa Inteligencia Artificial: estructura, producción y calidad informativa. Doxa Comunicación, 29, pp. 213-233. https://doi.org/10.31921/doxacom.n29a11

Ufarte Ruiz, M.J., Fieiras-Ceide, C., \& Túñez-López, M. (2020). La enseñanza-aprendizaje del periodismo automatizado en instituciones públicas: estudios, propuestas de viabilidad y perspectivas de impacto de la IA. Anàlisi: Quaderns de Comunicació i Cultura, 62, pp. 131-146. DOI: https://doi.org/10.5565/rev/analisi.3289

Valdiviezo-Abad, C. \& Bonini, T. (2019). Automatización inteligente en la gestión de la comunicación. Doxa Comunicación, 29, 169-196. https://doi.org/10.31921/doxacom.n29a9

Vállez, M. \& Codina, L. (2018). Periodismo computacional: evolución, casos y herramientas. El profesional de la información, 27 (4), pp. 759-768. https://doi.org/10.3145/epi.2018.jul.05

Van Dalen, A. (2012). The algorithms behind the headlines. Journalism Pratique 6(5-6), pp. 648-658. https://doi.org/10.1080/17512786.2012.667268

Wölker, A.\& Powell,TE. (2018). Algorithms in the newsroom? News readers perceived credibility and selection of automated journalism. Journalism. https://doi.org/10.1177/1464884918757072

Yanfang, W. (2019). Is Automated Journalistic Writing Less Biased? An Experimental Test of Auto-Written and Human-Written News Stories, Journalism Pratique, DOI: 10.1080 / 17512786.2019.1682940Balducci, B., \& Marinova, D. (2018). Unstructured data in marketing. Jour-nal of the Academy of Marketing Science, 46(4), pp. 557-590. 